\title{
Vehicle-Based Multicast Communication System for Live-Video
}

\author{
Yoseop Woo and Iksoo Kim ${ }^{1}$ \\ Dept. of Information and Telecommunication Eng. of Incheon National University \\ \{yswooo, iskim\}@incheon.ac.kr
}

\begin{abstract}
This paper ${ }^{2}$ proposes a vehicle-based communication system using multicast mechanism for live-video service. The vehicle-to-infrastructure (V2I) communication network (VICON) in this paper is composed of buffered-retransmission/switching/handover/ multicast agent (BRSHMA), some smart-Aps (SAP) and a number of vehicles. The BRSHMA and the SAPs perform handover using mapping table and an IP control packet, respectively. The BRSHMA supports multicast mechanism to solve the deficiency of VICON network bandwidth, takes charge of retransmission for packet lost/error and can reduce service delay time. Also, it can reduce the load of server and manages switching operation for the live-video streams from the server. We confirm the proposed mechanism provides smooth handover without reconnection to server and is added only a few bytes as overhead.
\end{abstract}

Keywords: V2I Communication, Multicast, Multimedia, Handover

\section{Introduction}

Vehicle-based communication, such as vehicle-to-infrastructure (V2I) and vehicle-tovehicle (V2V or ad-hoc mode), is very important and a hot issue with the development of vehicle industry. Their various application fields are logistic field for providing real time data as goods stock and status information of the vehicle, software updates, avoidance collision, road traffic information and many multimedia programs. Until now, the avoidance collision among various applications is main one in vehicle-based communication. Recently, however, multimedia service with the prevention of collision accident in V2V and V2I communication network is the one of the most popular services because wireless network has tremendous developed $[1,2,3,4]$.

The vehicle networks have some common problems that have a deficient network bandwidth(resources) and difficulty for a smooth handover just like wireless network including WLAN and ad-hoc network. The V2I communication network (VICON) suffers from cutoff connection to server when the vehicles are travelling. The vehicles disconnected have drawback to reconnect the server to sustain service. Thus many researches to solve these problems are being extensively studied. Especially, a deficient network bandwidth (resources) and the difficulty for a smooth handover due to the vehicles travelling and mobile nodes' movement have seriously affected for the vehicle-based network. Thus, many papers have studied multicast and web-caching mechanism to reduce the effect of a deficient

\footnotetext{
${ }^{1}$ Corresponding Author

2 This paper is extended and revised one that was presented at the 2013 IST conference[2].
} 
network bandwidth in multimedia service. And various studies about handover are proceeding actively in wireless network to support a seamless multimedia service $[4,5,6]$.

This paper presents the service mechanism that uses a small amount of network resources and reduces service delay. And it supports a smooth handover without reconnection to the server because of cutoff caused by vehicle's travelling. It adopts multicast technique, mapping table on the BRSHMA and an IP control packet issued by SAP to solve these problems.

The rest of this paper is as follows: Section 2 describes the structure and operations of V2I communication system using multicast and explains the operation of the components that is forming VICON network. Section 3 proposes algorithms to support a seamless live-video service. Section 4 deals with the results of simulation and finally, we discuss our conclusion.

\section{The Structure and Operation of Vehicle-to-Infrastructure Multicast Communication System}

The structure of the proposed V2I multicast communication network as shown in Fig. 1 is composed of a live-video server on Internet, a bufferedretransmission/switching/handover/multicast agent(BRSHMA), some smart-access points(SAP) with cache and a number of vehicles [5, 7].

The live-video server on Internet receives initial service requests, transmits live-video streams requested from vehicles through BRSHMA and SAP, but it does not work for the multicast mechanism including grouping.

The BRSHMA provides multicast mechanism for the same live-videos requested from vehicles. The BRSHMA and SAP with cache support retransmission for the lost or erroneous packets serviced just before that can occur while vehicles are driving. Also, they provide a smooth handover when vehicles are driving between SAPs in the VICON. And the coverage area of SAP is much wider than that of general AP in WLAN because the speed of vehicles is very fast compared pedestrians with mobile device in WLAN. Also, the BRSHMA provides switching to corresponding SAPs the successive live-video streams transmitted from the server. It has mapping table for the relation of SAP and vehicles managed, and switches to the corresponding SAP the successive streams using mapping table that will be described below. The BRSHMA performs multicast mechanism that creates a multicast group when a number of vehicles request the same live-video and then sends a multicast group address (MGA) to the vehicles. And it transmits multicasting streams to the SAPs that manage multicast grouped vehicles. Thus, the proposed VICON system has scarcely the possibility of multicasting packet duplication because the multicast mechanism is performed on the BRSHMA in VICON not the server in the Internet. Also, the BRSHMA may provide a partial rewind operation because it has buffer. The rewind operation and retransmission for lost packets, of course, is provided by unicast transmission unlike reference [8].

The SAP assigns IP address to the vehicles when they entry only into the VICON for the first time. It never assigns IP address again, though they move into the coverage of other SAPs after they are assigned it. It sends an IP control packet to BRSHMA and vehicles that move into its coverage area. The BRSHMA manages mapping table and updates it to provide a smooth handover using IP control packet that are sent from SAP. The IP control packet indicates the SAP of vehicle currently accessing and IP address assigned by the SAP accessed for the first time. The detailed explanation is in Figure 2. 


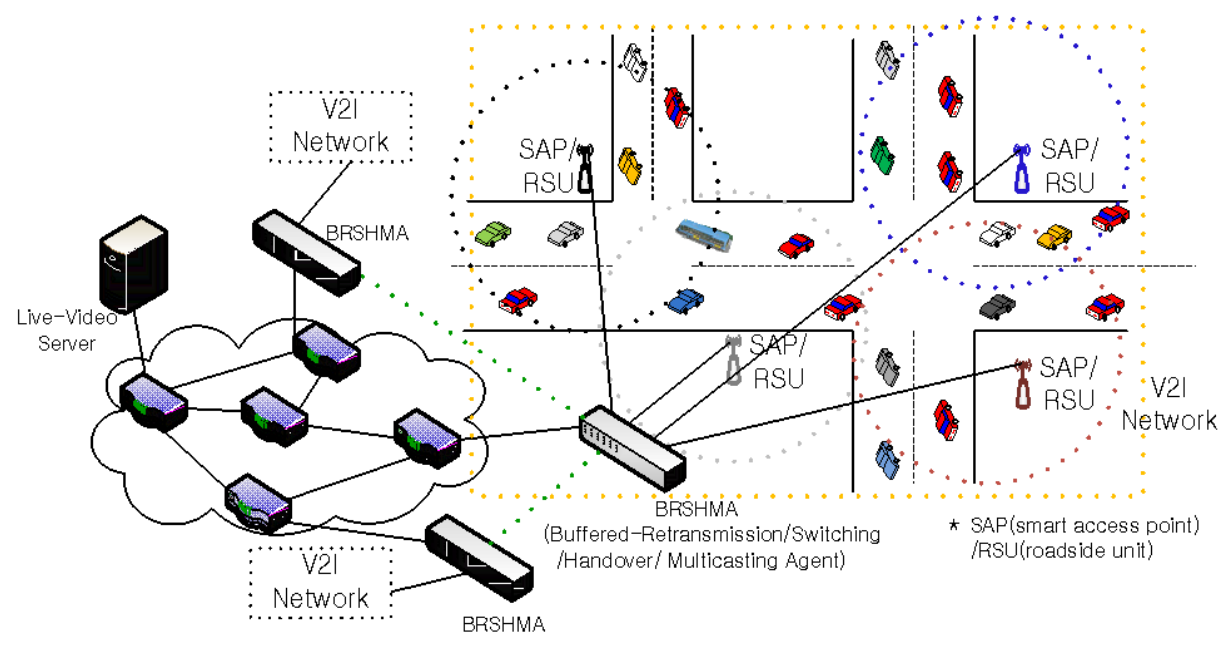

Figure 1. The structure of the proposed V2I network

\section{The mapping table on the BRSHMA and IP control packet of SAP}

The BRSHMA in VICON is the core component, performs multicast mechanism to reduce the load of live-video server, switching, handover and retransmission for lost/erroneous packet according to changing SAP according to vehicles travelling.

The BRSHMA and SAP perform smooth handover mechanism using mapping table and the control packet (a new IP header packet), respectively. The mapping table is divided into multicast one and unicast one. The format of mapping table is shown in Figure 2(a). It is composed of four fields-multicast bit(MB), MGA_\#, accessed AP_\# and assigned IP_\# field. The MB field is 1-bit long and indicates whether the serviced type of vehicle is multicast or unicast. The MB is set to 1 when the service request of vehicle is starting by other vehicle in VICON network, otherwise it is reset to 0(indicating unicast)

The MGA_\#(multicast group address number) field is 32-bits long(class-D address). The BRSHMA generates MGA_\# when more than 2 vehicles request the same live-video and sends it to the vehicles requested.

The accessed AP_\# field is 5-bits long that are changed according to the number of SAPs in VICON network. In this paper, let the number of SAPs is 31. Thus the range of SAP_\# is 00000 to 11110, and 11111 indicates initial state. The accessed SAP_\# indicates that accessed SAP_\# currently by vehicles, and is changed according to the vehicles travelling in VICON network.

The assigned IP_\# field is 32-bits long assigned by SAP accessed when the vehicle enters into the VICON network for the first time. This IP_\# never changes even if the vehicles are travelling among SAPs. 


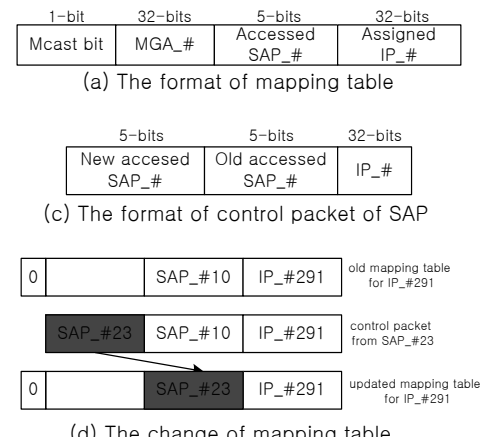

(d) The change of mapping table

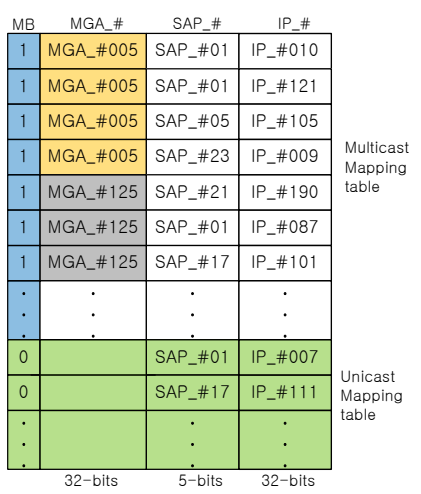

(b) The structure of mapping table on BRSHMA

Figure 2. The structure of mapping table on the BRSHMA and IP control packet format from the SAP

The structure of mapping table on the BRSHMA is shown Figure 2(b). The first 4-rows indicate IP_\#010 and 121 on the coverage of SAP_\#01, IP_\#105 accessed SAP_\#05 and IP_\#009 accessed SAP_\#23 are grouped MGA_\#005. Thus, it indicates the 4-vehicles are serviced the same live-video with MGA_\#005 and the BRSHMA transmits distributively live-video streams from server to the SAP_\#01, 05 and 23. And the 4-vehicles that have IP_\#010, 121, 105 and 009 join with MGA_\#005 for receiving multicast streams. Also, the shaded green portion of the mapping table shows unicast one(MB: 0 ).

The SAP generates control packet whenever vehicles enter within its coverage and the format of the control packet of SAP is shown Figure 2(c). The control packet is divided into 3 -fields that are composed of new accessed new accessed SAP_\#, old accessed SAP_\# and IP address(IP_\#) field. The SAP sends the control packet to the BRSHMA and the vehicles. The SAP assigns IP address to the vehicle only if it enters VICON network for the first time and this IP address never changes even if the vehicle are travelling among the SAPs. The control packet becomes 11111-01010-IP_\# when a specific vehicle enters SAP_\#10 in VICON network for the first time. The BRSHMA stores it on the mapping table and the vehicle can connect to server with assigned IP address(IP_\#291). The SAP_\#23 sends a control packet(10111-01010-IP_\#291) to BRSHMA when the vehicle is moved to SAP_\#23 and BRSHMA updates its mapping table old accessed SAP_\#(01010) into new accessed SAP_\#(10111). It is shown in Figure 2(d).

\section{Algorithm of V2I Multicast Communication System for Live-video service}

We describe the structure and operation of the proposed VICON network including BRSHA and SAP. As mentioned in Section 2, the BRSHMA is the core component in VICON network, and it takes charge of switching, multicast and handover mechanism as effective techniques for providing live-video service. In Section 3, to support effective livevideo service using multicast and handover for providing connectivity according to vehicles' driving in VICON, we present the algorithm that is composed of 2-parts as follows,

\section{Service algorithm in Vehicle-to-Infrastructure Network}

i) The vehicle $e_{i}$ requests accessing $S A P_{i}$ when vehicle $e_{i}$ entered the communication range of $S A P_{i}$ in VICON network for the first time

ii) The SAP $P_{i}$ assigns a SAP_\#i and IP address( IP_\#i) to the vehicle $i$ 
iii) The vehicle ${ }_{i}$ request live-video_\#i to server through the $S A P_{i}$ and BRSHMA

iv) The BRSHMA checks mapping table if the live-video_\#i is serviced already

v) If live-video_\#i is serviced with unicast(for vehicle ${ }_{k}$ )

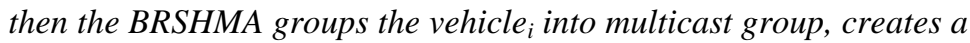
new multicast group address(MGA_\#i) for the live-video_\#i and sends MGA_\#i to the vehicle $e_{i}$ and vehicle ${ }_{k}$, and it fills out this in formation on its mapping table and goto step viii)

else if the BRSHMA checked vehicle $i$ is serviced already with MGA_\#i then the BRSHMA sends MGA_\#i to the vehicle $e_{i}$ and updates mapping table, and goto step vii) else the BRSHMA requests live-video_\#i to the live-video server

vi) The BRSHMA transmits live-video_\#i streams transmitted from the live-video server to vehicle $_{i}$ through unicast channel and it fills out vehicle in unicast portion of its mapping table

vii) The vehicle receives live-video_\#i streams transmitted from the BRSHMA through SAP $_{i}$

viii) The vehicle $e_{i}$ joins multicast group MGA_\#i to receive MGA_\#i streams, and then displays them

\section{Handover algorithm in Vehicle-to-Inf rastructure Network}

i) The vehicle ${ }_{i}$ requests accessing $S A P_{i}$ when vehicle ${ }_{i}$ entered the communication range of $S A P_{i}$ in VICON network for the first time

ii) The SAP ${ }_{i}$ sends control packet to the vehicle $e_{i}$ and BRSHMA

iii) The BRSHMA fills out this on its mapping table

iv) If the travelling vehicle $e_{i}$ accesses $S A P_{j}$ from $S A P_{i}$, then the $S A P_{j}$ sends its SAP no.(SAP_\#j) to the vehicle ${ }_{i}$ (SAP_\#j:IP address) and notifies it to the BRSHMA with SAP ${ }_{j}$ 's control packet(SAP_\#j: SAP_\#i:IP_\#i address)

v) The BRSHMA updates mapping table according to the $S A P_{j}$ 's control packet and the vehicle $e_{i}$ changes a part of its IP header $\left(S A P_{-} \# i \rightarrow S A P_{-} \# j\right)$ using the SAP's control packet

vi) If the vehicle does not receive a part of stream(stream $k$ ) transmitted from the BRSHMA according to the travelling from SAP_\#i to SAP_\#j

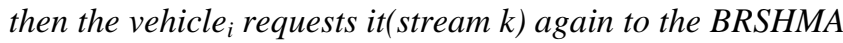

vii) The BRSHMA checks its mapping table whether the request of the vehicle ${ }_{i}$ is unicast or multicast group

viii) If the request is grouped for multicast then the BRSHMA sends MGA_\# to the vehicle

iv) The BRSHMA sends stream $k$ on its buffer again using unicast address to the vehicle ${ }_{i}$ on SAP-\#j : IP address_\#i)

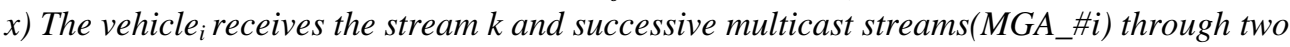
channels

\section{Result and Analysis of the Proposed System}

The results of simulation for the proposed system in VICON are shown in Figure 4. The environment of the simulation in VICON is $5 \mathrm{~km}$ straight road for 4-lane, the number of vehicles is different with the speed of them and the number of SAPs is different with the coverage of them, too. The simulation is performed according to the speeds of vehicles on the road $(20 \mathrm{~km} / \mathrm{h}, 60 \mathrm{k} / \mathrm{hm}$ and $100 \mathrm{~km} / \mathrm{h})$ and the time of driving of vehicle (15 minutes). The numbers of SAPs are 25, 17 and 13 that have the radius of coverage of SAP is $100 \mathrm{~m}$. And the reason using the speed of $20 \mathrm{~km} / \mathrm{h}, 60 \mathrm{~km} / \mathrm{h}$ and $100 \mathrm{~km} / \mathrm{h}$ is for indicating that the case of congested, normal condition and highway road. 
The Figure 4(a) shows the result of the comparison of data amount transmitted in network for the proposed handover mechanism and traditional one. The Figure 4(a) indicates that the comparison of network data amount between the proposed mechanism and traditional one for a smooth handover according to the speed of vehicle $(20 \mathrm{~km} / \mathrm{h}, 60 \mathrm{~km} / \mathrm{h}$ and $100 \mathrm{~km} / \mathrm{h})$ in VICON that has the coverage area of the SAP is $100 \mathrm{~m}$ for radius. The number of vehicles that passing the road $(5 \mathrm{~km})$ is different according to the speed of them and the rates of request for service are $10 \%$ to $70 \%$ of the vehicles. For $100 \mathrm{~m}$ of the coverage of the SAP, the performance of the proposed mechanism is improved about 36\%. The Figure 4(b) shows the result of the comparison of the number of necessary network channels for the multicast and unicast according to the rate (10\% to $70 \%)$ for multicast group when the service request rate is $50 \%$ of vehicles that are passing the road. It indicates the performance of the proposed mechanism according to the speed of vehicle in VICON network. The performance is improved $17.5 \%(47.5 \% 67.5 \%), 10 \%(37.5 \%, 57.5 \%)$ and $3.6 \%(21 . \% 40.5 \%)$ when the request rates for multicast due to the speed of vehicles $(20 \mathrm{~km} / \mathrm{h}, 60 \mathrm{~km} / \mathrm{h}$ and $100 \mathrm{~km} / \mathrm{h})$ are $20 \%$ (50\% and $70 \%$ ). Also, this paper is reduced control packet into 42 bits and the size of mapping table per one request into 70bits, and is simplified handover/multicast mechanism compared to reference paper 7.
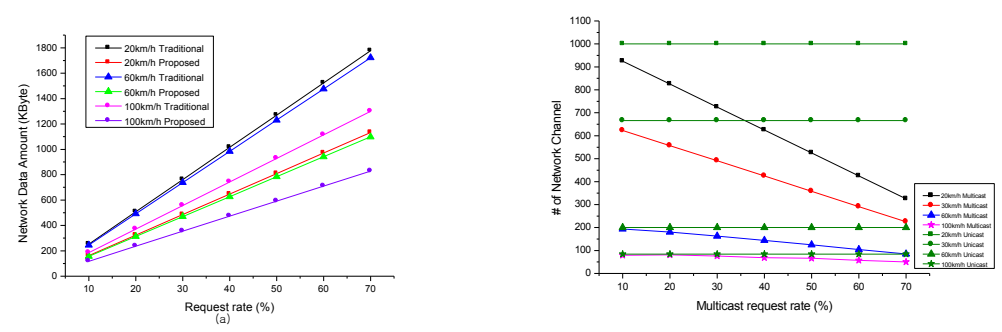

\section{Figure 4. The performance comparisons according to the rates of request for the speed of vehicles}

\section{Conclusion}

This paper supports a seamless live-video service using multicast technique in vehicle-toinfrastructure communication network (VICON). The mapping table on the BRSHMA and the control packet of SAP perform a smooth handover and their overheads are 70-bits per one request and 42-bits, respectively. Especially, the overhead for control packet is negligible compared with the video streams transmitted in VICON network. We confirm that the amount of network data transmitted for the proposed handover is improved about $36 \%$ compared to the traditional one and necessary numbers of channels are improved about $3.6 \%$ to $67.5 \%$ according to the rate of request and the speeds of vehicles.

\section{Acknowledgements}

This work was supported by the Incheon National University Research Grant in 2013. 


\section{References}

[1] G. Gehlen, E. Weiss and B. Walke, "Architecture of a vehicle communication gateway for media independent handover", Proc. of the $3^{\text {rd }}$ Int'l workshop on intelligent transportation, (2006), Hamburg, Germany.

[2] J. Choi, Y. Woo and I. Kim, "Vehicle-to-Infrastructured(V2I) Multicast Transmission for Live-Video Streams", Accepted from IST2013 conference, (2013) June 27-30; Bali, Indonesia.

[3] A. Mostafa, A. Vegni, R. Singoria, T. Oliveira, T. Little and D. Agrawal, "A V2X-based approach for reduction of delay propagation in Vehicular Ad-Hoc Networks", MCL Technical Report No. 07-18-2011, (2011).

[4] U. Dwivedi, A. Rajawati and A. Upadhyay, "Characterize an approach to improve performance of multimedia services over vehicular ad-hoc network", J. of Information System and Communication. ISSN: 0976-8742 \& E-ISSN: 0976-8750, vol. 3, (2012).

[5] S. Lee and I. Kim, "Multimedia Service using Equi-loaded Cache in Wireless Network", J. of Korean Institute of Informaton Technology, (2012) March.

[6] R. Bolla, R. Rapuzzi and M. Repetto, "User-Centric Mobility for Multimedia Communication: Experience and Evaluation from a Live Demo", J. of Networks, vol. 7, no. 1, (2012) January.

[7] Y. Woo and I. Kim, "Multicasing System for a Seamless Live-Multimedia in WLAN", Submitted for EURASIP J.on Wireless Communications and Networking.

[8] Y. -W. Chen and L. -R. Han, "A Novel Broadcasting Strategy for Interactive Video-on-Demand Services, Tamsui Oxford Journal of Mathematical Sciences, (2005), Aletheia University.

\section{Authors}

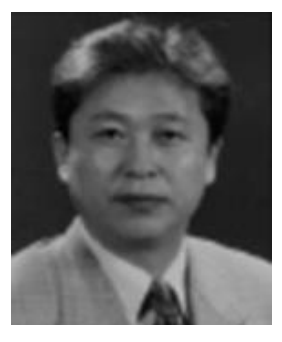

\section{Iksoo Kim}

August,1985: A Ph.D. degrees in Electronics Engineering from Dongguk University, Korea

The present: A professor in department of Information and telecommunication engineering, University of Incheon

Major area of interest: Multicast, Multimedia System, Ad-hoc Network.

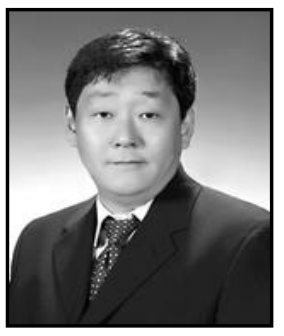

\section{Yoseop Woo}

February, 1992: A Ph.D. degrees in Electronics Engineering from Hanyang University, Korea.

The present: A professor in department of Information and telecommunication engineering University of Incheon

Major area of interest: Data processing Korean language, Network System, Database. 
International Journal of Multimedia and Ubiquitous Engineering Vol.8, No.5 (2013) 